\title{
EDUCAÇÃO CONTINUADA EM UM HOSPITAL MUNICIPAL: RELATO DE EXPERIÊNCIA
}

\section{CONTINUING EDUCATION IN A MUNICIPAL HOSPITAL: EXPERIMENT REPORTS}

\section{EDUCACIÓN CONTINUA EN UN HOSPITAL MUNICIPAL: RELATO DE LA EXPERIENCIA}

Lorrany Fontenele Moraes da Silva ${ }^{1}$, Marcela Rangel de Almeida², Pedro Martins Lima Neto ${ }^{3}$, Simony Fabíola Lopes Nunes ${ }^{4}$, Lívia Maia Pascoal ${ }^{5}$, Francisco Dimitre Rodrigo Pereira Santos 6

\section{RESUMO}

Objetivo: Relatar a vivência discente no desenvolvimento de um Programa de Educação Continuada para profissionais da equipe de enfermagem de um hospital municipal. Método: Relato de experiência, realizado em um hospital da rede pública com 51 profissionais, dentre eles enfermeiros, técnicos de enfermagem e auxiliares de enfermagem participaram da educação continuada desenvolvida por discentes de um projeto de extensão. A implementação da educação continuada foi dividida em quatro etapas: diagnóstico, intervenção, avaliação e entrega de certificados aos participantes. Resultados: Dos 51 profissionais, a maioria era do sexo feminino e técnico de enfermagem, em relação ao grau de satisfação e organização o programa foi avaliado como ótimo, e a principal dificuldade encontrada foi a sobrecarga de trabalho. Conclusão: A experiência possibilitou constatar que a elaboração e desenvolvimento de um Programa de Educação Continuada é desafiadora, entretanto, impulsiona a transformação da assistência de enfermagem e possibilita a aproximação dos discentes com o campo de prática.

Descritores: Educação continuada; Capacitação profissional; Enfermagem; Período pós-operatório.

\section{ABSTRACT}

Objective: to report the experience of students in the development of a Continuing Education Program for professionals of a nursing team from a municipal hospital. Method: experience report produced in a public hospital with 51 professionals, including nurses, nursing technicians and nursing auxiliaries who participated in the continuing education developed by students as a part of an extension project. The implementation of continuing education was divided into four stages: diagnostic, intervention, evaluation and delivery of certificates to participants. Results: most of the 51 professionals were women and nursing technicians. Regarding the degree of satisfaction and organization, the program was evaluated as great, and the main found difficulty was the work overload. Conclusion: the experience made it possible to realize that the elaboration and development of a Continuing Education Program is challenging. However, it drives the transformation of nursing care and allows the students to approximate to the field of practice. Descriptors: Continuing Education; Professional training; Nursing; Postoperative Care.

\section{RESUMEN}

Objetivo: relatar la experiencia discente en el desarrollo de un Programa de la Educación Continua para profesionales del equipo de enfermería de un hospital municipal. Método: relato de la experiencia, consumado entre noviembre de 2015 a mayo de 2016, en un hospital público del ayuntamiento de Imperatriz/MA. 51 profesionales, entre otros, enfermeros, técnicos de enfermería y auxiliares de enfermería participaron en la educación continua desarrollada por discentes de un proyecto de extensión. La implementación de la educación continua fue dividida en cuatro etapas: diagnostico, intervención, evaluación y entrega de certificados para los participantes. Resultados: de los 51 profesionales la mayoría eran mujeres y técnicos de enfermería, en relación al grado de satisfacción y organización el programa fue valorado como optimo, y la principal dificultad encontrada fue la sobrecarga de trabajo. Conclusión: la experiencia posibilitó verificar que la elaboración y el desarrollo de un programa de educación continua es desafiador, sin embargo estimula la transformación de la asistencia de la enfermería y hace posible la aproximación de los discentes al campo de la práctica.

Descriptores: Educación Continua; Capacitación Profesional; Enfermería; Periodo Posoperatorio.

${ }^{1}$ Enfermeira. Universidade Federal do Maranhão. ${ }^{2}$ Enfermeira. Universidade Federal do Maranhão. ${ }^{3}$ Fisioterapeuta. Mestre. Universidade Federal do Maranhão. ${ }^{4}$ Enfermeira. Mestre. Universidade Federal do Maranhão. ${ }^{5}$ Enfermeira. Doutora. Universidade Federal do Maranhão. ${ }^{6}$ Fisioterapeuta. Mestre. Unidade de Ensino Superior do Sul do Maranhão e Universidade Estadual do Tocantins.

\section{Como citar este artigo:}

Silva LFM, Almeida MR, Neto PML, et al. Educação continuada em um hospital municipal: relato de experiência. Revista de Enfermagem do Centro Oeste Mineiro. 2020;10:e2713. [Access___]; Available in:___ DOI: http://doi.org/10.19175/recom.v10i0.2713 


\section{INTRODUÇÃO}

Ao longo do desenvolvimento socioeconômico-político do país, observou-se a importância da educação para os profissionais de saúde, por estes necessitarem, constantemente, de aperfeiçoamento, durante a formação da vida profissional. A educação continuada para esses profissionais tem como objetivo a qualificação, o aperfeiçoamento do exercício profissional com maior segurança e produtividade, por meio da aquisição de novos conceitos e da reformulação de práticas já existentes ${ }^{(1)}$.

A terminologia relacionada à educação continuada e educação permanente precisa ser esclarecida para uma melhor compreensão da escolha do método. A educação continuada compõe um processo educativo e atua com a finalidade de oferecer ao profissional uma reflexão sobre o seu desempenho profissional e ajustá-lo diante das necessidades nos serviços de saúde ${ }^{(2)}$, enquanto que a educação permanente trabalha na perspectiva da transformação, participa do desenvolvimento das ações de ensino em serviço, considera as singularidades, necessidades de formação e desenvolvimento para o trabalho em saúde, fortalecendo a atenção integral a saúde ${ }^{(3)}$.

O ponto culminante da educação continuada é fazer dos profissionais de saúde formadores de opinião, para construírem um saber crítico e qualificar, cada vez mais, o cuidado. Para isso, é necessário compreender e acolher todo o processo educativo, que fornece ao profissional, instrumentos de mudança e inovação, por meio dos conhecimentos adquiridos. Toda essa reciclagem incentivada pela educação continuada tem o propósito de gerar uma transformação no indivíduo e no ambiente de trabalho ${ }^{(4)}$.

Nas instituições hospitalares, os profissionais de enfermagem desenvolvem inúmeras tarefas com alto grau de exigências e responsabilidades, além das atividades de gestão, tornando-se fundamental a adesão destes às práticas educativas, em resposta à construção continua do saber e às demandas de serviço, a fim de melhorar o desempenho profissional ${ }^{(5)}$.

Portanto, acreditando que a aquisição de mais informações possa melhorar a competência dos profissionais de enfermagem para desenvolver suas responsabilidades e elevar a motivação ao trabalho, programas de educação continuada podem contribuir assim para a sistematização do processo de trabalho ${ }^{(6)} . \mathrm{Em}$ contrapartida, nota-se a existência de uma relutância quanto à adesão ao processo educativo, pois a práxis da dissociação entre profissional assistencial e profissional educador é muito forte e imperativa, revelando uma lacuna na busca pela atualização do conhecimento. A partir dessa problematização, no presente artigo, objetivouse relatar a vivência discente, no desenvolvimento de um Programa de Educação Continuada para profissionais da equipe de enfermagem de um hospital municipal.

\section{MÉTODO}

Trata-se de um estudo descritivo, do tipo relato de experiência, realizado entre os meses de novembro de 2015 a maio de 2016, em um hospital da rede pública do município de Imperatriz, Maranhão, Brasil. As ações desse relato de experiência fazem parte do projeto de extensão intitulado: Capacitação sobre Assistência de Enfermagem no Pós-operatório de Cirurgia Torácica e Abdominal Alta, vinculado à PróReitoria de Extensão - PROEX/UFMA, edital № 16/2015, e ao Hospital Municipal de Imperatriz.

Objetivou-se com o projeto proporcionar a atenção à saúde, por meio de atividades educativas, promoção de cuidados respiratórios aos pacientes em pós-operatório de cirurgia tóraco-abdominais, assistidos no Hospital Municipal de Imperatriz. É desenvolvido com a participação interdisciplinar, sendo composto por quatro docentes e 19 discentes do curso de Enfermagem e Medicina da Universidade Federal do Maranhão, além do apoio de coordenadores e profissionais das diversas áreas do hospital. Perante essa interinstitucionalização, a tríade ensino-pesquisa-extensão atua, de forma integrativa, aproximando o discente para vivência da prática profissional, pelo incentivo para a aplicação dos conhecimentos adquiridos em sala de aula. Além disso, desperta o interesse pela produção científica e necessidade constante de atualização.

A educação continuada foi implementada, no hospital, direcionada para a equipe de enfermagem que atua na clínica cirúrgica, com o objetivo de auxiliar na segurança do paciente, melhorando $o$ atendimento prestado aos pacientes, no período pós-operatório, diminuindo o número de complicações respiratórias e tempo de internação.

A sua composição deu-se em quatro etapas. A primeira etapa, o diagnóstico, consistiu na análise da necessidade local e de relatos da 
equipe. As enfermarias da clínica cirúrgica realizam, em média, 4,1 procedimentos cirúrgicos diários. O setor é composto por 26 leitos, distribuídos em 07 enfermarias. A equipe de saúde é composta por 07 médicos ( 05 cirurgiões gerais e 02 cirurgiões plásticos), 06 enfermeiros, 21 técnicos de enfermagem, 25 auxiliares de enfermagem, 01 fisioterapeuta e 01 nutricionista.

A segunda etapa constou da intervenção, a partir da elaboração e realização de palestras, por ser um recurso eficiente e de fácil aplicação. Estas foram efetivadas uma vez ao mês, no horário matutino, na sala de reunião do posto da clínica cirúrgica, com uma média de 50 minutos de duração. A seleção dos temas abordados foi discutida com a participação dos gestores e profissionais de saúde do hospital, juntamente com equipe docente e discente do projeto. Os temas abordados, durante o período de execução, foram: Sinais Vitais, Semiologia Cardiorrespiratória, Oxigenoterapia, Dor e Orientações de Posicionamento no PósOperatório, Autocuidado Após Alta e Reanimação Cardiorrespiratória. Participaram do projeto os enfermeiros, técnicos e auxiliares de enfermagem da clínica cirúrgica do hospital que atuam no pósoperatório de cirurgia geral.

A terceira etapa norteou a avaliação do projeto com a interação dos participantes, realizada ao final de cada palestra. Foi construído um instrumento com questões abertas e fechadas para avaliar a eficácia dos temas abordados para assistência de enfermagem, a perspectiva dos profissionais de saúde a cerca do projeto e a influência das capacitações na realidade vivenciada pelos profissionais, no campo de prática; dados sobre o grau de satisfação da capacitação graduado em excelente, ótimo, bom, ruim e péssimo; questões sobre as dificuldades enfrentadas para participar da capacitação; propostas para aumentar a adesão da equipe multiprofissional e sugestões de temas. O instrumento ainda constava de dados sociodemográficos, formação acadêmica e tempo de exercício profissional.

A quarta e última etapa, considerou a entrega de certificados com carga horária de 30 horas, para os profissionais participantes que atingiram, no mínimo, quatro presenças ao decorrer do projeto. A capacitação foi composta por seis módulos, na qual o controle das presenças foi realizado, por meio de frequências devidamente assinadas pelos ouvintes.

A pesquisa é parte de um projeto guardachuva, aprovado pelo Comitê de Ética em Pesquisa da Universidade Federal do Maranhão (CEPUFMA), com o parecer de número 629.315.

\section{RESULTADOS E DISCUSSÕES}

A vivência no Programa de Educação Continuada contribuiu de maneira significativa para a formação acadêmica, sendo possível analisar os resultados e compreender os erros e acertos encontrados ao longo do programa.

Dos 51 profissionais participantes, $88,23 \%$ eram do sexo feminino, $86,28 \%$ eram técnicos de enfermagem, $80,40 \%$ eram adultos de meia idade $^{(7)}$ (23 a 56 anos), com média de idade de 38,80 anos. $O$ tempo de exercício profissional mais recorrente foi de 6 a 11 anos, representando $54,90 \%$ (Tabela 1 ).

Tabela 1 - Perfil sociodemográfico dos participantes do Programa de Educação Continuada. Imperatriz, Maranhão, Brasil, 2016

\begin{tabular}{llc}
\hline \hline Variável & $\mathbf{N}$ & $\%$ \\
\hline Sexo & 06 & 11,77 \\
$\quad$ Masculino & 45 & 88,23 \\
$\quad$ Feminino & & 19,60 \\
Idade & 10 & 80,40 \\
$\quad$ Adulto-Jovem & 41 & 86,28 \\
$\quad$ Adulto de Meia Idade & & 9,80 \\
Profissão & 44 & 3,92 \\
$\quad$ Técnicos de enfermagem & 05 & 21,57 \\
$\quad$ Enfermeiros & 02 & 54,90 \\
$\quad$ Auxiliares de enfermagem & & 7,84 \\
Exercício profissional & 11 & 15,69 \\
$1-5$ anos & 28 & 04 \\
$6-11$ anos & 08 & \\
$12-17$ anos & & \\
$18-23$ anos
\end{tabular}

Fonte: dados da pesquisa. 
Verificou-se que a maioria dos participantes era do sexo feminino, fundamentando a expressiva feminização no meio dos profissionais de saúde. Esse predomínio é decorrente da herança cultural, onde, no final do século XIX, a profissionalização feminina desenvolveu-se vinculada à imagem da mulher ao cuidar, ao servir, ao educar e, nesse contexto, a enfermagem tornou-se a primeira profissão feminina universitária no $\mathrm{Brasil}^{(8)}$, sem deixar de citar o seu outro nível de formação como técnico em enfermagem ${ }^{(9)}$.

Dos profissionais da equipe de enfermagem, os técnicos demonstraram grande interesse na participação do programa, sendo a maioria do público-alvo, diferentemente, dos enfermeiros que pouco, aderiram às capacitações. Esses achados corroboram com um estudo realizado com profissionais de enfermagem de um hospital em Maringá, Paraná, Brasil, no qual constatou-se que apenas uma pequena parte da amostra participava, frequentemente, de um Programa de Educação(10).
Analisando o tempo de exercício profissional, metade dos participantes tem entre 6-11 anos de atuação, sendo necessário o constante contato com as evoluções dos conhecimentos em saúde. Vale lembrar que os assuntos abordados no Programa de Educação Continuada eram direcionados para a realidade vivenciada pelos profissionais de enfermagem, buscando uma melhor integração com a teoria e prática. Pesquisa realizada em Porto Alegre, Rio Grande do Sul, Brasil, com o objetivo de compreender como os enfermeiros aplicavam os conhecimentos adquiridos em sua vivência acadêmica, demonstrou falhas na integração entre teoria e prática influenciada pelo tempo de formação e pela realidade do local de trabalho ${ }^{(11)}$.

Em relação ao grau de satisfação e organização do Programa de Educação Continuada, $41,18 \%$ avaliaram como ótimo a qualidade das capacitações, o mesmo foi considerado por $47,06 \%$ dos participantes sobre a configuração organizacional (Tabela 2).

Tabela 2 - Grau de satisfação e organização do Programa de Educação Continuada. Imperatriz, Maranhão, Brasil, 2016

\begin{tabular}{lll}
\hline \hline Variável & $\mathbf{N}$ & $\%$ \\
\hline Qualidade das capacitações & 17 & 33,33 \\
Excelente & 21 & 41,18 \\
Ótimo & 13 & 25,50 \\
Bom & 00 & 00,00 \\
Ruim & 00 & 00,00 \\
Péssimo & & 19,60 \\
Configuração organizacional & 10 & 47,06 \\
Excelente & 24 & 31,38 \\
Ótimo & 16 & 00,00 \\
Bom & 00 & 01,96 \\
Ruim & 01 & \\
Péssimo & & \\
\hline \hline
\end{tabular}

Fonte: dados da pesquisa.

O programa foi qualificado como ótimo, pois os temas ministrados foram voltados para $o$ cuidado e a segurança do paciente, sendo de grande relevância para a prática clínica assistencial, isso confirma o interesse e a aceitação dos profissionais em participar da educação continuada. Estudos abordam que esse interesse pode contribuir para o desenvolvimento de competências dos profissionais como alternativa para propor mudanças e transformações, dentro do espaço de trabalho e na própria organização, recriando uma nova forma de assistir os clientes e proporcionando a transição do tecnicismo para o desenvolvimento de capacidade crítica e participação ativa na qualidade do serviço em beneficio dos usuários $^{(6,12)}$.

No decorrer da primeira etapa, as discentes objetivaram estabelecer um vínculo com a equipe a ser trabalhada, tanto com profissionais da saúde, como com os profissionais do setor administrativo, pois além de conquistar a confiabilidade do staff, as mesmas tiveram que estudar a realidade e necessidade local, por meio de observações da rotina hospitalar e coleta de relatos da equipe. Ao ser deparadas com a 
diferença entre a visão teórica e a realidade hospitalar existente, esse processo foi visto como um desafio por parte das discentes, o que gerou ansiedade e muita expectativa.

Decorrente do processo inicial, os docentes e as discentes propuseram à equipe de saúde que elucidassem as dificuldades mais comuns na rotina, resultando em alguns pontos a serem abordados. Diante disso, as discentes puderam repensar a forma pedagógica que os temas seriam ministrados, abandonando uma pedagogia hierárquica e tradicional, centralizada na figura do professor, para aderir a uma forma reflexiva, crítica e dinâmica, onde foi induzido o diálogo entre $o$ palestrante $e$ os participantes, favorecendo a construção de saberes em ambas as vias.

Por meio da avaliação das palestras, que foi possível com aplicação do instrumento, deu-se espaço à exteriorização da experiência individual, profissional e pessoal de cada colega, valorizando assim, a recíproca do saber. Nesse momento, os profissionais avaliariam o trabalho desenvolvido até então. Essa etapa foi de suma importância para as discentes, pois puderam mensurar o nível de aceitabilidade e satisfação do programa, favorecendo a aquisição de domínio no planejamento, organização e ministração de temas importantes para a equipe, visando à formação de enfermeiros críticos e ativos no processo de cuidar.

A vivência entre discentes, docentes e profissionais do hospital foi importante para o progresso da integração entre universidade e serviço, além de promover essa aproximação com o campo profissional, estimula o senso crítico tanto dos discentes quanto dos profissionais, para a construção de conhecimento, possibilitando um aperfeiçoamento profissional. Em consonância com essa vivência, um estudo realizado com docentes e discentes, no âmbito do ensino e aprendizagem, destacaram que práticas, nesse contexto, promovem nos discentes uma passagem da consciência ingênua para a consciência crítica, guiando a construção do perfil do profissional de saúde e na formação profissional|(13).

Os principais desafios encontrados pela equipe executora foram ausência de um espaço físico bem estruturado, dificuldade na adequação do horário dos profissionais para a participação da educação continuada, visto a impossibilidade de não poderem abandonar seu posto de trabalho para participar dos encontros.

As dificuldades mais relatadas pelos profissionais foram sobrecarga de trabalho e divulgação ineficiente dos horários das atividades, com $62,75 \%$ e $23,53 \%$ respectivamente (Tabela 3 ).

Tabela 3 - Principais dificuldades relatadas para participação no Programa de Educação Continuada. Imperatriz, Maranhão, Brasil, 2016

\begin{tabular}{llc}
\hline \hline Variável & $\mathbf{N}$ & $\mathbf{\%}$ \\
\hline Principais Dificuldades & 32 & $65,75 \%$ \\
Sobrecarga de trabalho & 12 & $23,53 \%$ \\
Divulgação ineficiente dos horários das atividades & 03 & $3,90 \%$ \\
$\begin{array}{l}\text { Divulgação ineficiente dos propósitos e importância das atividades } \\
\text { de educação continuada }\end{array}$ & 03 & $3,90 \%$ \\
Não liberado no momento em que as atividades são realizadas & 01 & $1,90 \%$ \\
Desconhecimento das atividades & & \\
\hline \hline
\end{tabular}

Fonte: dados da pesquisa.

Constatou-se que a sobrecarga de trabalho foi a maior dificuldade encontrada pelos participantes, fato que pode estar associado à falha no processo de gestão e gerência e/ou deficiência no quantitativo de recursos humanos do hospital. Com isso, é importante que haja a modernização e inovação no processo administrativo, promovendo uma descentralização hierárquica, incentivando uma maior autonomia no processo de trabalho, diminuição da burocracia e, consequentemente, redução da sobrecarga de trabalho, para que não comprometa a segurança e bem-estar do paciente ${ }^{(14-15)}$.

As longas jornadas de trabalho interferem, diretamente, na qualidade da assistência, sendo um fator preponderante na sobrecarga de trabalho associado ao acúmulo de atividade, número reduzido de profissionais e sobrecarga de funções. Esse contexto produz reflexos negativos 
não somente para os pacientes, como também para os profissionais, gerando doenças ocupacionais, dentre elas o estresse, em decorrência do efeito cumulativo das atividades do ambiente de trabalho ${ }^{(15)}$.

A divulgação ineficiente foi relatada por parte dos profissionais como um ponto negativo da equipe de organização, sendo um fator crucial na diminuição da participação desses profissionais. No entanto, ao longo do desenvolvimento do Programa de Educação Continuada, o trabalho de divulgação foi realizado em conjunto com os mais diversos setores do hospital, sendo realizada, principalmente, por meio de distribuição de panfletos e cartazes e por meio das redes sociais, com o intuito de atingir a ampla maioria dos profissionais.

Apesar das dificuldades encontradas pelas equipes de enfermagem em participar das capacitações, é significativa essa experiência no ambiente de trabalho como um espaço de criação e manutenção do conhecimento, proporcionando a aprendizagem coletiva e, consequentemente, profissionais capacitados. Referindo-se a isso, a literatura aponta que, apesar das dificuldades, os profissionais precisam entender a real importância da educação continuada, pois é uma oportunidade para capacitação, além de propiciar melhoria na qualidade da assistência e segurança ao paciente, visto que mobiliza também outros membros da equipe multidisciplinar e não somente a enfermagem ${ }^{(16)}$.

Como alternativa de vivenciar as necessidades dos profissionais frente à assistência, os participantes, ao responderem o questionário, sugeriram temas para serem desenvolvidos pelo projeto em capacitações posteriores, estes foram divididos nos seguintes eixos temáticos e contemplaram: doenças crônicas, feridas e curativos, saúde do trabalhador, doenças infecciosas, urgência e emergência e assistência de enfermagem.

Como a educação continuada é um processo que objetiva a integração do indivíduo à sociedade, ressalta-se que o modo como o processo de trabalho é problematizado pode proporcionar mudanças positivas na organização do trabalho, bem como nas práticas profissionais, favorecendo a equipe multiprofissional a criação de perfis direcionados para as necessidades da população, de acordo com cada realidade regional e nível de complexidade.

O estudo reforça a necessidade da constante renovação e adesão ao processo de educação continuada, para o aprimoramento de profissionais devidamente qualificados. Isso reflete, na prática acadêmica, como um ponto crucial na construção de conhecimento e formação de opiniões, contribuindo para o desenvolvimento da enfermagem como ciência.

\section{CONCLUSÃO}

A experiência vivenciada, neste relato, foi enriquecedora e de fundamental importância para a construção do saber, tanto para os profissionais de saúde quanto para a vida acadêmica. Foi possível perceber que a implementação de um Programa de Educação Continuada é desafiadora, contudo, uma ação necessária, uma vez que a adesão desse processo educativo impulsiona a transformação da assistência de enfermagem, por meio do aprimoramento e atualização de conhecimentos técnico-científicos.

Por se tratar de um estudo do tipo relato de experiência, não foram utilizados instrumentos validados, o que pode constituir uma limitação deste estudo. Acredita-se que, pela importância do tema, investigações sistemáticas devem ser realizadas, para acrescentar mais conhecimento do assunto.

A equipe participante do estudo contribuiu, de maneira significativa, para a vivência acadêmica, direcionando o estudo para as deficiências, necessidades e dificuldades encontradas dentro do local de trabalho, fortalecendo, assim, o vínculo e a interinstitucionalização entre universidadehospital. Portanto, destaca-se a importância do envolvimento discente em processos de educação continuada, pois possibilita a aproximação dos discentes com o campo de prática para atuarem futuramente, permitindo cultivar esse novo olhar na assistência de enfermagem.

\section{REFERÊNCIAS}

1- Coswosk ED, Santa Rosa CG, Caldeira AB, Silva NCR, Rocha JM. Continuing education for the health professional in Health waste management. RBAC 2018;50(3):288-96. DOI: $10.21877 / 2448-$ 3877.201800645

2- Bezerra ALQ, Queiroz ES, Weber J, Munari DB. $O$ processo de educação continuada na visão de enfermeiros de um hospital universitário. Rev Eletr Enferm. 2012;14(3):618-25. DOI: 10.5216/ree.v14i3.12771

3- Cardoso MMVN, Ferreira RGS. Continuing or permanent education: Common goal with the predominance of specificities towards the 
teaching-learning process. Rev Saúde Desenvolv. 2014 [citado em 2020 fev 22]; 5(3):126-36. Available in: https://www.uninter.com/revistasaude/index.ph $\mathrm{p} /$ saudedesenvolvimento/article/view/280/203 4- Lazzari DD, Schmidt N, Jung W. Educação continuada em unidade de terapia intensiva na percepção de enfermeiras. Rev Enferm UFSM 2012 [citado em 10 set 2017]; 2(1):88-96. Available in: https://periodicos.ufsm.br/reufsm/article/view/4 $592 / 3130$

5- Soares MI, Resck ZMR, Camelo SHH, Terra FS. Gerenciamento de recursos humanos e sua interface na sistematização da assistência de enfermagem. Rev Enfermería Global 2016 [citado em 23 fev 2020]; 42:353-64. Available in: http://scielo.isciii.es/pdf/eg/v15n42/pt_administr acion3.pdf

6- Salum NC, Prado ML. Continuing education in the development of competences in nurses. Texto Contexto-Enferm. 2014;23(2):301-8. DOI: 10.1590/0104-070720140021600011

7- Torres TL, Camargo BV, Bousfield ABS. Social stereotypes of elderly for different age groups. Psic: Teor e Pesq. 2016;32(1): 209-18. DOI: 10.1590/0102-37722016012114209218

8- Neves GBC, Andreto LM, Oliveira CR, Figueira MCS. Opinião dos enfermeiros sobre educação permanente em um hospital públic. Rev Enferm UFPE 2016;10(5):1625-34. DOI: 10.5205/reuol.9003-78704-1-SM.1005201608

9- Monteiro RP, Jung W, Lazzari DD, Nascimento ERP, Dalamaria JM. The professional transition process from the perspective of nursing technicians who have become nurses. Rev Eletr Enferm. 2014;16(4):777-86. DOI: 10.5216/ree.v16i4.24129

10- Pereira FMV, Malaguti-Toffano SE, Silva AM, Canini SRMS, Gir E. Adherence to standard precautions of nurses working in intensive care at a university hospital. Rev Esc Enferm USP 2013;47(3):686-93. DOI: 10.1590/S0080623420130000300023

11- Lazzari DD, Jacobs LG, Jung W. Humanização da assistência na enfermagem a partir da formação acadêmica. Rev Enferm UFSM 2012;2(1):116-24. DOI: 10.5902/217976923705 12- Gonçalves LC, Cortez EA, Calvacanti ACD, Cosme FSM, Valente GSC. Educação permanente sob o olhar de profissionais da estratégia de saúde da família. Rev Enferm UFPE 2014;8(supl 1):23906 DOI: $10.5205 /$ reuol.5927-50900-1SM.0807supl201426
13- Rodrigues SG, Neves MGC. Formative assessment: Experiences in active methodology of teaching and learning from the teachers and students perspectives. Comun Ciênc Saúde 2015 [citado em 24 fev 2020]; 26(3/4):105-14. Available in:

http://bvsms.saude.gov.br/bvs/periodicos/ccs_a rtigos/avaliacao_formativa.pdf

14- Lima A, Nicolato F, Dutra H, Bahia M, Farah $B$. A educação permanente na gestão da atenção primária de saúde no sistema único de saúde. Rev Enferm UFPE 2015 [citado em 13 jun 2017]; 9(supl 4):8135-45. Available in: https://periodicos.ufpe.br/revistas/revistaenfer magem/article/view/10569/11507

15- Novaretti MCZ, Santos EV, Quitério LM, Daud-Gallotti RM. Sobrecarga de trabalho da enfermagem e incidentes e eventos adversos em pacientes internados em UTI. Rev Bras Enferm. 2014;67(5):692-9. DOI: 10.1590/00347167.2014670504

16- Souza LP, Lima MG. Continuous education in intensive care unit-icu: Literature review. J Health Biol Sci. 2015 [citado em 24 fev 2020]; 3(1):39-45. Available in: https://periodicos.unichristus.edu.br/jhbs/article /view/137/101

Nota: Pesquisa originada do Projeto de Extensão intitulado Capacitação sobre assistência de enfermagem no pósoperatório de cirurgias torácicas e abdominais.

Recebido em: 26/03/2018

Aprovado em: 09/04/2020

Endereço de Correspondência:

Universidade Federal do Maranhão, Campus Bom Jesus, Coordenação do Curso de Medicina, Av. da Universidade, S/N, Bairro Dom Afonso Felipe Gregory, CEP 65915-240, Imperatriz, Maranhão

E-mail:pedro.martins@ufma.br 